01,02

\title{
Об одном нелинейном эффекте в теории сверхпроводимости
}

\author{
() С.О. Гладков
}

Московский авиационный институт (национальный исследовательский университет) МАИ, Москва, Россия

E-mail: sglad51@mail.ru

Поступила в Редакцию 15 ноября 2018 г.

В окончательной редакции 30 июня 2019 г.

Принята к публикации 8 июля 2019 г.

Из решения гидродинамических уравнений и уравнений Максвелла показано, что внешнее квазиоднородное магнитное поле приводит к появлению вторичного электрического поля, являющемуся результатом проявления нелинейного эффекта по магнитному потенциалу А. Доказано, что это поле существует в области глубиной $\frac{\delta}{2}$, где $\delta-$ лондоновская глубина проникновения. Приведена оценка скорости гидродинамического потока.

Ключевые слова: уравнения Максвелла, глубина проникновения, магнитное поле, электрическое поле, нелинейный эффект.

DOI: 10.21883/FTT.2019.11.48397.317

\section{1. Введение}

Вопрос, на котором нам хотелось бы сейчас остановиться, относится к общим физическим свойствам теории сверхпроводимости, основанной не на микроскопической теории БКШ (Бардин, Купер, Шифер), а на языке макроскопических уравнений гидродинамики и уравнений Максвелла, также вполне естественными, как, например, полярность вектора электрического поля $\mathbf{E}(t)=\mathbf{E}(-t)$, и аксиальность магнитного $\mathbf{H}(t)=\mathbf{H}(-t)$, являющимися их неотъемлемой характеристикой.

Речь будет идти о нелинейном эффекте, связанным с возможным появлением вторичного квазиоднородного электрического поля, которое, как увидим, всегда существует и также является неотъемлемым свойством в явлении сверхпроводимости. В силу нелинейности этого эффекта область существования вторичного электрического поля будет определяться величиной $\frac{\delta}{2}$, которая диктует максимальную глубину проникновения потока электрического поля в толщу проводника. Надо сказать, что мы не нашли никакой содержательной информации, касающейся изложения этого вопроса, в огромном количестве оригинальных статей и монографий (см., к примеру [1-7]), посвященных как микро- так и макроскопической теории сверхпроводимости. Заметим еще к слову, что сами уравнения Лондонов являются только следствием уравнений Максвелла (см. ниже) в предположении, что коллективное движение электронов по какой-то физической причине представляет собой обычный гидродинамический поток. Как сейчас увидим, подобный подход приводит к весьма любопытному выводу о том, что в квазистатическом приближении (электрическое и магнитное поля независимы), внешнее магнитное поле, в котором находится металл при температурах сверхпроводящего перехода, должен привести к появлению вторичного электрического поля, проявляющему себя лишь в виде нелинейного эффекта по магнитному потенциалу А (см. ниже), что вовсе не противоречит уравнениям Максвелла.

Возможность существования этого поля приводит к ряду довольно любопытных эффектов, о которых мы поговорим далее.

\section{2. Решение задачи}

Будем считать, что газ заряженных частиц движется c некоторой гидродинамической скоростью $\mathbf{v}=\mathbf{v}(\mathbf{r}, t)$ сквозь толщу металла под действием приложенных электрического и магнитного полей, и пусть $\rho_{0}=\frac{d m}{d V}-$ плотность потока, где $d m$ - элемент массы движущихся частиц, а $\rho_{1}=\frac{d q}{d V}-$ плотность их электрического заряда, где $d q$ - элемент заряда, $d V$ - элемент объема. Тогда в соответствии с уравнением Лоренца можно записать, что

$$
\rho_{0} \dot{\mathbf{v}}=\rho_{1}\left(\mathbf{E}+\frac{[\mathbf{v} \times \mathbf{V}]}{c}\right) .
$$

Известно, что для гидродинамического потока полная производная от скорости по времени есть просто субстанциональная производная [8], то есть

$$
\dot{\mathbf{v}}=\frac{\partial \mathbf{v}}{\partial t}+(\mathbf{v} \cdot \nabla) \mathbf{v}
$$

В результате из (1) следует уравнение, которое ранее ни в одном из известных нам источников не приводилось

$$
\rho_{0}\left(\frac{\partial \mathbf{v}}{\partial t}+(\mathbf{v} \cdot \nabla) \mathbf{v}\right)=\rho_{1}\left(\mathbf{E}+\frac{\mathbf{v} \times \mathbf{H}}{c}\right) .
$$

Далее, согласно уравнениям Максвелла [7] имеем

$$
\operatorname{div} \mathbf{D}=4 \pi \rho_{1},
$$




$$
\begin{gathered}
\operatorname{div} \mathbf{B}=0, \\
\operatorname{rot} \mathbf{E}=-\frac{1}{c} \frac{\partial \mathbf{B}}{\partial t}, \\
\operatorname{rot} \mathbf{H}=\frac{1}{c} \frac{\partial \mathbf{D}}{\partial t}+\frac{4 \pi}{c} \mathbf{j},
\end{gathered}
$$

где плотность тока

$$
\mathbf{j}=\rho_{1} \mathbf{v}
$$

(некоторые другие чисто практические приложения теории ЭМ поля можно найти, например, в работах [9-11]).

Из уравнений (4) и (5) следуют известные определения для полей $\mathbf{E}$ и $\mathbf{B}$ через магнитный потенциал $\mathbf{A}$ и электрический $\varphi$ в виде [7]

$$
\left\{\begin{array}{l}
\mathbf{B}=\operatorname{rot} \mathbf{A}, \\
\mathbf{E}=-\frac{1}{c} \frac{\partial \mathbf{A}}{\partial t}-\nabla \varphi .
\end{array}\right.
$$

Подставив (8) в уравнение (2), найдем

$$
\rho_{0}\left(\frac{\partial \mathbf{v}}{\partial t}+(\mathbf{v} \cdot \nabla) \mathbf{v}\right)=\left(-\frac{1}{c} \frac{\partial \mathbf{A}}{\partial t}-\nabla \varphi+\frac{[\mathbf{v} \times \operatorname{rot} \mathbf{A}]}{c}\right) .
$$

Слагаемое $[\mathbf{v} \times \operatorname{rot} \mathbf{A}]$ преобразуем с помощью формулы

$$
[\mathbf{v} \times \operatorname{rot} \mathbf{A}]=\nabla_{\mathbf{A}}(\mathbf{v} \cdot \mathbf{A})-(\mathbf{b} \cdot \nabla) \mathbf{A} .
$$

Где значок А внизу у оператора „набла“ означает, что его действие распространяется только на вектор А. После подстановки (10) в уравнение (9) находим

$$
\begin{aligned}
\rho_{0}\left(\frac{\partial \mathbf{v}}{\partial t}+(\mathbf{v} \cdot \nabla) \mathbf{v}\right) \\
\quad=\rho_{1}\left(-\frac{1}{c} \frac{\partial \mathbf{A}}{\partial t}-\nabla \varphi+\frac{1}{c}\left(\nabla_{\mathbf{A}}(\mathbf{v} \cdot \mathbf{A})-(\mathbf{v} \cdot \nabla) \mathbf{A}\right)\right) .
\end{aligned}
$$

В результате элементарной перегруппировки слагаемых будем иметь

$$
\begin{aligned}
& \frac{\partial}{\partial t}\left(\rho_{0} \mathbf{v}+\frac{\rho_{1}}{c} \mathbf{A}\right)+(\mathbf{v} \cdot \nabla)\left(\rho_{0} \mathbf{v}+\frac{\rho_{1}}{c} \mathbf{A}\right)+\rho_{1} \nabla \\
& \times\left(\varphi-\frac{(\mathbf{v} \cdot \mathbf{A})}{c}\right)+\frac{\rho_{1}}{c}([\mathbf{A} \times \operatorname{rot} \mathbf{v}]+(\mathbf{A} \cdot \nabla) \mathbf{v}) \\
& -\frac{\mathbf{A}}{c}\left(\frac{\partial \rho_{1}}{\partial t}+\left(\mathbf{v} \cdot \nabla \rho_{1}\right)\right)-\mathbf{v}\left(\frac{\partial \rho_{0}}{\partial t}+\left(\mathbf{v} \cdot \nabla \rho_{0}\right)\right)=0 .
\end{aligned}
$$

В силу уравнения электронейтральности

$$
\frac{\partial \rho_{1}}{\partial t}+\operatorname{div}\left(\rho_{1} \mathbf{v}\right)=0
$$

и уравнения непрерывности

$$
\frac{\partial \rho_{0}}{\partial t}+\operatorname{div}\left(\rho_{0} \mathbf{v}\right)=0
$$

имеем из (11)

$$
\begin{aligned}
& \frac{\partial}{\partial t}\left(\rho_{0} \mathbf{v}+\frac{\rho_{1}}{c} \mathbf{A}\right)+(\mathbf{v} \cdot \nabla)\left(\rho_{0} \mathbf{v}+\frac{\rho_{1}}{c} \mathbf{A}\right) \\
& \quad+\left(\rho_{0} \mathbf{v}+\frac{\rho_{1}}{c} \mathbf{A}\right) \operatorname{div} \mathbf{v}+\rho_{1} \nabla_{\mathbf{A}}\left(\varphi-\frac{(\mathbf{v} \cdot \mathbf{A})}{c}\right)=0 .
\end{aligned}
$$

Благодаря независимости параметров $\mathbf{v}$ и $\varphi$ из получаются два следующих уравнения

$$
\rho_{0} \mathbf{v}=-\frac{\rho_{1}}{c} \mathbf{A},
$$

и

$$
\varphi-\frac{(\mathbf{v} \cdot \mathbf{A})}{c}=S(t),
$$

где $S(t)$ - некоторая функция времени.

Заметим, что решение (16) не противоречит уравнениям Максвелла. Действительно, если взять градиент от обеих частей уравнения (16), то получим

$$
-\nabla \varphi-\frac{1}{c} \frac{\partial \mathbf{A}}{\partial t}=-\frac{1}{c} \nabla(\mathbf{v} \cdot \mathbf{A})-\frac{1}{c} \frac{\partial \mathbf{A}}{\partial t} .
$$

Откуда следует, что $\mathbf{E}=-\frac{1}{c} \nabla(\mathbf{v} \cdot \mathbf{A})-\frac{1}{c} \frac{\partial \mathbf{A}}{\partial t}$. Взяв отсюда ротор, и воспользовавшись определением (8), приходим к известному уравнению $\operatorname{rot} \mathbf{E}=-\frac{1}{c} \frac{\partial \mathbf{B}}{\partial t}$.

\section{3. Обобщение уравнения Лондонов}

Остановимся теперь на некоторых следствиях соотношений (15) и (16).

Из уравнения (15) видно, что $\mathbf{v}=-\frac{\rho_{1}}{c \rho_{0}} \mathbf{A}$. Подставляя это решение в (7), а его, в свою очередь, в уравнение (6), получаем

$$
\operatorname{rot} \mathbf{H}=\frac{1}{c} \frac{\partial \mathbf{E}}{\partial t}-\frac{4 \pi \rho_{1}^{2}}{\rho_{0} c} \mathbf{A} .
$$

Используя, наконец, решения (8) и пользуясь уравнением калибровки ЭМ поля

$$
\operatorname{div} \mathbf{A}+\frac{1}{c} \frac{\partial \varphi}{\partial t}=0,
$$

приходим в итоге к волновому уравнению вида

$$
\Delta \mathbf{A}-\frac{1}{c^{2}} \frac{\partial^{2} \mathbf{A}}{\partial t^{2}}-\frac{4 \pi \rho_{1}^{2}}{\rho_{0} c} \mathbf{A}=0 .
$$

Надо заметить, что уравнение (19) отличается от уравнения Лондонов, которое, как известно [7], имеет вид

$$
\Delta \mathbf{B}-\frac{4 \pi e^{2} n_{s}}{m c} \mathbf{B}=0 .
$$

И дело здесь вовсе не во второй производной по времени, а в плотностях $\rho_{0}$ и $\rho_{1}$. В самом деле, если учесть, что $\rho_{0}=m n_{s}$, а $\rho_{1}=e n_{s}$, где $n_{s}=\frac{d N_{s}}{d V}-$ концентрация носителей, $d N_{s}$ их количество в объеме $d V$, то после 
взятия операции „ротор“ от уравнения (26) в квазистатическом приближении, опустив вторую производную по времени, получим

$$
\Delta \mathbf{B}-\frac{4 \pi e^{2}}{m c} \mathbf{B}-\frac{4 \pi e^{2}}{m c} \mathbf{B}\left[\nabla n_{s} \times \mathbf{A}\right]=0,
$$

где последнее слагаемое учитывает возможное проявление неоднородности потока электронов. Для однородного потока имеет место уравнение Лондонов (20). Соотношение (15) при этом позволяет написать, что

$$
\operatorname{div} \mathbf{v}=-\frac{e}{m c} \operatorname{div} \mathbf{A}
$$

и в квазистатическом приближении согласно условию калибровки ЭМ потенциалов (18) $\operatorname{div} \mathbf{A}=0$ мы приходим к уравнению несжимаемости

$$
d i v \mathbf{v}=0
$$

В рассматриваемом нами статическом случае вторая производная по времени исчезает, и из уравнения (19) получим

$$
\Delta \mathbf{A}-\frac{\mathbf{A}}{\delta^{2}}=0
$$

где параметр лондоновской длины определяется соотношением $\frac{1}{\delta^{2}}=\frac{4 \pi e^{2} n_{s}}{m c^{2}}$.

Выбирая произвольный малый участок поверхности проводника $l$ такой, что он удовлетворяет условию $l \ll \lambda$, где $\lambda$ - длина волны внешнего магнитного поля, можно считать что в перпендикулярном поверхности металла направлении, которое мы принимаем за ось $z$, направленную вверх, из уравнения (23) следует единственное физически разумное решение, затухающее при $z \rightarrow-\infty$

$$
\mathbf{A}=\mathbf{A}_{0} e^{\frac{z}{\delta}}
$$

где $\mathbf{A}_{0}$ - значение векторного потенциала на поверхности проводника при $z=0$.

В соответствии с (7) и (16) для плотности тока будем иметь

$$
\mathbf{j}=-\frac{\rho_{1}^{2}}{\rho_{0} c} \mathbf{A}=-\frac{e^{2} n_{s}}{m c} \mathbf{A} .
$$

Уравнение (25) напоминает уравнение Лондонов [1-7], однако, в (25) мы говорим о плотности тока, а не о токе. Действительно, если в (25) подставить концентрацию носителей тока в виде $n_{s}=\frac{d N_{s}}{d V}$, то получим

$$
\int \mathbf{j} d V=-\frac{e^{2}}{m c} \int n_{s} \mathbf{A} d V .
$$

В силу же решения (24) отсюда следует, что $\int \mathbf{j} d V=\mathbf{J} \delta$, а потому, в однородном случаем немедленно приходим к уравнению Лондонов

$$
\mathbf{J}=-\frac{e^{2} N_{s}}{m c \delta} \mathbf{A}
$$

Заметим также, что если от уравнения (25) взять операцию ротор, то получим уравнение

$$
\operatorname{rot} \mathbf{j}=-\frac{e^{2} N_{s}}{m c} \mathbf{B}
$$

которое было получено Л.Д. Ландау, но другим способом, а его вывод приведен в монографии [7].

\section{4. Оценка глубины проникновения электрического поля в сверхпроводник}

Проанализируем теперь соотношение (16). Подставляя в него решение (15) и полагая $S=0$, найдем

$$
\varphi=-\frac{e^{2} A^{2}}{m c^{2}}
$$

Это означает, что для вторичного электрического поля, действующего строго вдоль оси $z$, имеем

$$
E_{z}=-\frac{d \varphi}{d z}=\frac{e A_{0}^{2}}{m c^{2}} \frac{d}{d z} e^{\frac{2 z}{\delta}}=\frac{2 e A_{0}^{2}}{m c^{2} \delta} e^{\frac{2 z}{\delta}} .
$$

Из решения (29) следует, что квазиоднородное электрическое поле может проникать внутрь сверхпроводника только на глубину $\frac{\delta}{2}$ (где лондоновская длина $\delta$ определена в уравнении (23)), а на поверхности сверхпроводника оно имеет конечное значение, равное

$$
E_{z}(0)=\frac{2 e A_{0}^{2}}{m c^{2} \delta}
$$

Формула (30) отвечает на поставленный вначале статьи вопрос, и указывает на существование вторичного электрического поля на поверхности сверхпроводника, которое является просто его свойством.

\section{5. Оценка скорости гидродинамического потока}

Чтобы вычислить скорость гидродинамического потока, то первое предположение, которое, казалось бы, появляется, это воспользоваться уравнением (2) Однако, если подставить в него соотношениями (15) и (16), то мы, как это и должно быть, приходим к тождеству. Сказанное означает, что для вычисления скорости гидродинамического потока $\mathbf{v}$ нам следует воспользоваться иными соображениями, не используя уравнение (2).

Для его получения обратимся к уравнению (6) и соотношениям (15), (16). Действительно согласно (15) $\mathbf{v}=-\frac{e}{m c} \mathbf{A}$ или $\mathbf{A}=-\frac{m c \mathbf{v}}{e}$, а согласно (16) $\varphi=\frac{(\mathbf{v} \cdot \mathbf{A})}{c}=-\frac{m}{e} v^{2}$. Поэтому благодаря равенствам $\mathbf{B}=\mu \mathbf{H}, \mathbf{D}=\varepsilon \mathbf{E}$, где $\mu, \varepsilon-$ соответственно магнитная 
и диэлектрическая проницаемости, из уравнения (6) получаем

$$
\text { rotrot } \mathbf{A}=\text { graddiv } \mathbf{A}-\Delta \mathbf{A}=\frac{\mu \varepsilon}{c} \frac{\partial \mathbf{E}}{d t}+\frac{4 \pi \mu}{c} \rho_{1} \mathbf{v}
$$

Но $\mathbf{E}=-\frac{1}{c} \frac{\partial \mathbf{A}}{\partial t}-\nabla \varphi$ и благодаря условию калибровки $\operatorname{div} \mathbf{A}+\frac{1}{c} \frac{\partial \varphi}{\partial t}=0$ легко находим отсюда

$$
\frac{\mu \varepsilon}{c^{2}} \frac{\partial^{2} \mathbf{A}}{\partial t^{2}}-\Delta \mathbf{A}=\frac{4 \pi \mu}{c} \rho_{1} \mathbf{v}
$$

Подставив сюда $\mathbf{A}=-\frac{m c \mathbf{v}}{e}$, получаем искомое уравнение на скорость потока

$$
\Delta \mathbf{v}-\frac{1}{u_{0}^{2}} \frac{\partial^{2} \mathbf{v}}{\partial t^{2}}-\frac{\mathbf{v}}{\delta^{2}}=0
$$

где $u_{0}=\frac{c}{\sqrt{\mu \varepsilon}}$. Полученное уравнение позволяет вычислить пространственно - временное распределение гидродинамической скорости. Как из него видно, если скорость потока направлена вдоль оси $x$, то стационарное и чисто физическое формальное решение вблизи поверхности сверхпроводника будет

$$
v_{x}(z)=v_{F} e^{\frac{z}{\delta}}
$$

где $v_{F}-$ скорость гидродинамического потока вблизи его поверхности, которая, очевидно, должна совпадать со скоростью нормальных электронов, представляющую из себя просто скорость Ферми. Напомним, что направление оси $z$ выбрано от поверхности вовнутрь тела.

Рассмотрим теперь уравнение (32) для реального практического случая, когда проводник представляет собой проволоку радиуса $R$. Тогда для скорости $\mathbf{v}=\left(v_{x}(r), 0,0\right)$ в статическом случае в системе цилиндрических координатах уравнение (32) примет вид

$$
\frac{1}{r} \frac{d}{d r} r \frac{d v_{x}}{d r}-\frac{v_{x}}{\delta^{2}}=0 .
$$

Его решение есть

$$
v_{x}(r)=C J_{0}\left(i \frac{r}{\delta}\right),
$$

где $C$ - константа интегрирования, а $J_{0}(i x)-$ функция Бесселя нулевого порядка от мнимого аргумента.

Из граничного условия $v_{x}(R)=v_{F}$ следует, что $C=\frac{v_{F}}{J_{0}\left(i \frac{R}{\delta}\right)}$. Поэтому решение будет

$$
v_{x}(r)=v_{F} \frac{J_{0}\left(i \frac{r}{\delta}\right)}{J_{0}\left(i \frac{R}{\delta}\right)}
$$

Отсюда легко сделать вывод о том, что скорость потока на оси проволоки оказывается такой

$$
v_{x}(0)=\frac{v_{F}}{J_{0}\left(i \frac{R}{\delta}\right)}
$$

Пользуясь асимптотикой модифицированной функции Бесселя первого типа [12]

$$
J_{0}(i x) \approx \frac{e^{x}}{\sqrt{2 \pi x}}
$$

при $x \gg 1$, благодаря условию $R \gg \delta$ имеем из (37)

$$
v_{x}(0) \approx v_{F} \frac{e^{-\frac{R}{\delta}} \sqrt{\delta}}{\sqrt{2 \pi R}} .
$$

Как видно из решения (38), скорость потока быстро убывает с расстоянием, что вполне соответствует реальности, и не противоречит теории Гинзбурга-Ландау.

Стоит еще раз подчеркнуть, что предложенный выше подход опирался на два главных предположения.

Классическая теория сверхпроводимости может быть сконструирована с помощью теории электромагнитного поля Максвелла и уравнений гидродинамики. Во всяком случае серьезных разногласий приведенного выше подхода с теорией Гинзбурга-Ландау (сокращенно Г-Л) мы не заметили.

Действительно, если внимательно посмотреть на формулу (30), то легко увидеть следующее. Поскольку электрическое поле на поверхности сверхпроводника ведет себя как $E_{z}(0)=\frac{2 e A_{0}^{2}}{m c^{2} \delta}$, а глубину проникания $\delta$ можно представить через параметр порядка теории $Г-Л \psi$ в виде

$$
\delta=\sqrt{\frac{m c^{2}}{4 \pi e^{2} n_{s}}}=\sqrt{\frac{m c^{2}}{4 \pi e^{2}|\psi|^{2}}}=\sqrt{\frac{m c^{2} T_{c}}{4 \pi e^{2} n_{0}\left(T_{c}-T\right)}},
$$

то это как раз и означает, что возникающее электрическое поле должно зависеть от параметра порядка линейно в соответствии с формулой

$$
E_{z}(0)=\frac{4 \sqrt{\pi} e^{2} A_{0}^{2}}{m c^{3} \sqrt{m}}|\psi| .
$$

Чтобы убедиться в том, что этот эффект вполне может быть обнаружен экспериментально, оценим численное значение величины поля на поверхности сверхпроводника, исходя из соотношения (40).

Полагая по порядку величины, что заряд электрона и его масса есть $e \sim 10^{-10} \mathrm{CГC}, m \sim 10^{-27} \mathrm{~g}$, а скорость света в вакууме составляет $c \sim 10^{10} \frac{\mathrm{cm}}{\mathrm{s}}$, в Гауссовой системе единиц немедленно получаем, что

$$
E_{z}(0) \sim 10^{-8} A_{0}^{2}|\psi| .
$$

Как видим, эффект оказывается вполне ощутимым, если речь идет не о самой точке сверхпроводящего перехода $T_{s}$, а о температурах лишь ее порядка, поскольку в этом случае $|\psi| \sim \sqrt{n_{s}} \sim 10^{11} \mathrm{~cm}^{-3}$.

В связи со сказанным следует также отметить, что предложенное выше описание имеет одно, на наш взгляд, вполне оправданное преимущество, которое довольно сильно отличает его от классических теорий, поскольку речь идет о предсказании эффекта нелинейного выталкивания из толщи сверхпроводника электрического поля, о чем ранее в известных нам публикациях не упоминалось. 


\section{6. Заключение}

1. Показана принципиальная возможность проявления нелинейного эффекта, связанная с появлением вторичного электрического поля в сверхпроводнике, происхождение которого индуцируется внешним магнитным полем, и приведена его связь с параметром порядка в теории Гинзбурга-Ландау.

2. Дана оценка области существования вторичного электрического поля вблизи поверхности сверхпроводника, и найдена глубина его проникновения в толщу проводника, составляющая ровно половину лондоновского параметра длины.

3. Показано, что лондоновская глубина проникновения квазиоднородного магнитного поля в сверхпроводник является естественным следствием уравнений Максвелла и уравнений гидродинамики, а не отличительной чертой сверхпроводимости.

4. Приведено аналитическое выражение для распределения скорости гидродинамического потока $v_{s}$, которую можно описать с помощью формул (33) и (36).

\section{Конфликт интересов}

Авторы заявляют, что у них нет конфликта интересов.

\section{Список литературы}

[1] Дж. Шриффер. Теория сверхпроводимости. Наука, М. (1970). $311 \mathrm{c.}$

[2] М. Тинкхам. Введение в сверхпроводимость. Атомиздат, М. (1980). 312 c.

[3] А.В. Свидзинский. Пространственно-неоднородные задачи теории сверхпроводимости. Наука, М. (1982). 422 с.

[4] M. Tinkham. Introduction to superconductivity. 2nd ed. Mc Graw-Hill (1996). 356 p.

[5] В.В. Шмидт. Введение в физику сверхпроводников. МЦНMO, M. (2000). $416 \mathrm{c}$.

[6] N.B. Kopnin. Theory of nonequilibrium superconductivity. Clarendon Press (2001). 421 p.

[7] Л.Д. Ландау, Е.М. Лифшиц. Электродинамика сплошных сред. Наука, М. (2004). Т. 8. 620 с.

[8] Л.Д. Ландау, Е.М. Лифшиц. Гидродинамика. Наука, М. (2004). T. 6. 742 c.

[9] С.О. Гладков. ЖТФ 85, 7, 138 (2015).

[10] С.О. Гладков, С.Б. Богданова. Радиотехника и электроника. 62, 7, 632 (2017).

[11] С.О. Гладков, С.Б. Богданова. Изв. вуз. Физика. 61, 1, 94 (2018).

[12] Г. Бейтмен, А. Эрдейи. Высшие трансцендентные функции. Наука, М. (1974). Т. 2. 295 с.

Редактор Т.Н. Василевская 